\title{
Single- Crystal Sapphire Optical Fiber Sensor Instrumentation
}

\section{Annual Report}

DOE Award Number: DE-FC26-99FT40685

Reporting Period Start Date: 1 October 2000

Reporting Period End Date: 30 September 2001

Issue Date: 31 October 2001

Principal Authors: A. Wang, G. Pickrell, and R. May

Submitted by: Center for Photonics Technology

Bradley Department of Electrical and Computer Engineering Virginia Tech

Blacksburg, VA 24061-0111

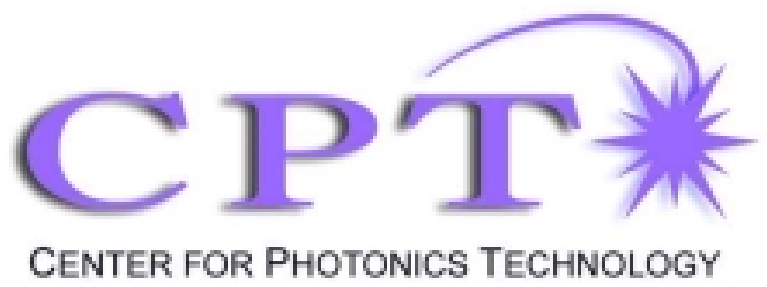




\section{Disclaimer}

"This report was prepared as an account of work sponsored by an agency of the United States Government. Neither the United States Government nor any agency thereof, nor any of their employees, makes any warranty, express or implied, or assumes any legal liability or responsibility for the accuracy, completeness, or usefulness of any information, apparatus, product, or process disclosed, or represents that its use would not infringe privately owned rights. Reference herein to any specific commercial product, process, or service by trade name, trademark manufacturer, or otherwise does not necessarily constitute or imply its endorsement, recommendation, or favoring by the United States Government or any agency thereof. The views and opinions of authors expressed herein do not necessarily state or reflect those of the United States Government or any agency thereof." 


\begin{abstract}
Testing results of a Broadband Polarized-Light Interferometric (BPLI) high temperature sensor is presented in this report. The state of polarization of the broadband incident light is modulated by the high birefringence of the sapphire disk used as the sensing element and becomes a wavelength-encoded signal, which is detected by an Optical Spectrum Analyzer (OSA) and then is processed by a computer, an internally developed algorithm is employed to directly calculate gap changes between two optical path between two orthogonal linear polarizations of light in a sapphire phase retarder, its phase retardation changes with temperature. The great advantages of this sensor are its simplicity and longterm stability in harsh environment. The system has been laboratory successfully tested up to $1600^{\circ} \mathrm{C}$.
\end{abstract}

\title{
Introduction
}

In the last report, Polarized-Light Interferometric Sensor (PLIS) was further optimized to improve its performance for larger temperature range, knowing that it is hard to fabricate high resolution and good repeatability sensors with commercial available products, and also some other disadvantages and limitations by comparing intensity measurement scheme with spectrum measurement scheme in optical sensor design, a new sensing scheme: Broadband Polarized-Light Interferometric (BPLI) high temperature sensing was presented, which is spectrum measurement based. From long term run point of view, this spectrum measurement configured system offers higher measurement resolution and the better measurement repeatability than intensity-based sensors, this kind of sensor guarantees that encoded spectrum data is not corrupted by intensity fluctuations in the test signal.

Based on some fundamental experiments results from BPLI system in the last report, this report will present some experiment results with certain improvements, which were obtained from optimization of experiment setups and a different optical source modulation method. Main works in this report will be the following: 
1. For calibration purpose, temperature-acquiring subsystem are designed to obtain real-time temperature values, which makes it possible to obtain gap value vs. temperature curves for the sensing system.

2. A sensing tube prototype is design to optimize the experiment setup. With sapphire protection tube and alumna extension tube, a 2 meter-long sensing tube is assembled and tested under higher temperature environment, and reflection mode scheme is tried instead of previous transmission mode in sensing tube design.

3. Optical source is modulated by rectangular waveform signals instead of sinusoidal waveform signal, to reduce blackbody radiation.

4. Performance of sensing system evaluation. Temperature range from $20^{\circ} \mathrm{C}$ up to $1600^{\circ} \mathrm{C}$ is tested; repeatability and stability are improved compared with results in last report. 


\section{Executive Summary}

A new temperature sensor configuration has been developed and tested based on a Broadband Polarized Light Interferometric Sensor (BPLIS). The sensor utilizes the temperature dependence of the birefringence of a single crystal sapphire sensing element to uniquely determine the temperature. This sensor system has been tested up to $1600^{\circ} \mathrm{C}$ and has shown excellent resolution, and repeatability. The system appears quite close to being ready to move to the preparation for field testing. The demonstrated resolution is at least as good as the resolution of the B type thermocouple used to calibrate this system. This resolution has been shown to be about $1{ }^{\circ} \mathrm{C}$.

The sensor probe has been ruggedized to improve the performance in the extremely harsh environment of the coal gasifier by utilizing single crystal sapphire elements. The sensor probe consists of an outer and inner single crystal sapphire tube approximately $25 \mathrm{~mm}$ overall outside diameter. Previous corrosion experiments have shown single crystal sapphire to have very good corrosion resistance in the coal slag in laboratory testing. The outer and inner sapphire tubes in the new design give a combined thickness of the single crystal sapphire protection tubes of approximately $6 \mathrm{~mm}$. The reflecting prism has been fabricated out of single crystal zirconia, a material with a melting point even higher than single crystal sapphire. The single crystal sapphire tubes have been connected to a high purity dense alumina extension tube approximately 1.5 meters long, allowing the probe to be able to penetrate the approximately 1 meter thick gasifier wall.

The entire prototype system including the single crystal sapphire probe, zirconia prism, alumina extension tube, optical components and signal processing hardware and software have shown excellent performance in the laboratory experimentation as the data presented in this report will document. 


\section{Table of Contents}

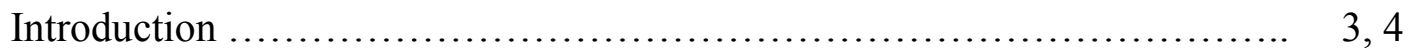

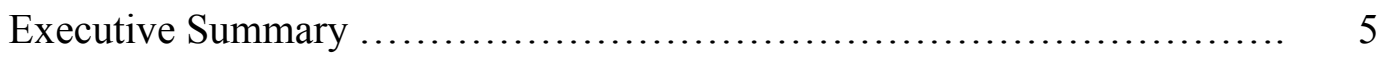

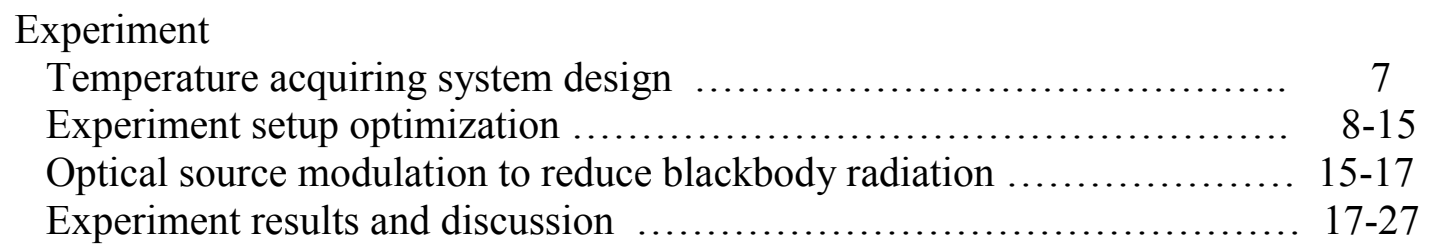

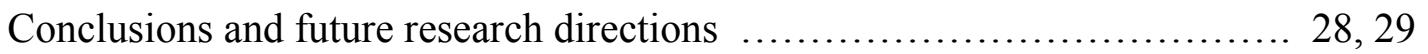




\section{EXPERIMENT}

\section{Temperature acquiring system design}

All experimental results shown in last report are mostly relations between thickness of sapphire disk vs. time, which is indirectly related to real time temperature values, our finial goal is to get the direct relation between temperature and thickness, to do so, a temperature acquiring subsystem is needed. In such a subsystem, real time temperature values from B type thermal coupler will be input into computer with $\mathrm{A} / \mathrm{D}$ card, this temperature value will be one to one related to thickness of sapphire disk by software communication between temperature acquiring subsystem and sapphire disk thickness measurement subsystem.

One DPi32-C24 temperature meter from Omega ${ }^{\circledR}$ is used to collect temperature values from B-type thermal couple, its RS-232 serial digital communication port is used to communicated with computer directly. By employing Visual_Basic, a graphical user interface (GUI) is designed to display the real-time temperature values; each of these values is uniquely corresponding to one gap value, these gap values are difference between two orthogonal linear polarized lights propagating path in the sensing element, as shown in Figure1, the gap value is 20.10391 micrometer at temperature $1181.0^{\circ} \mathrm{C}$.

With this temperature acquirement subsystem, the performance of the designed temperature sensing system, such as repeatability, accuracy compared to thermal couple and temperature resolution and etc, can be evaluated in detail. 


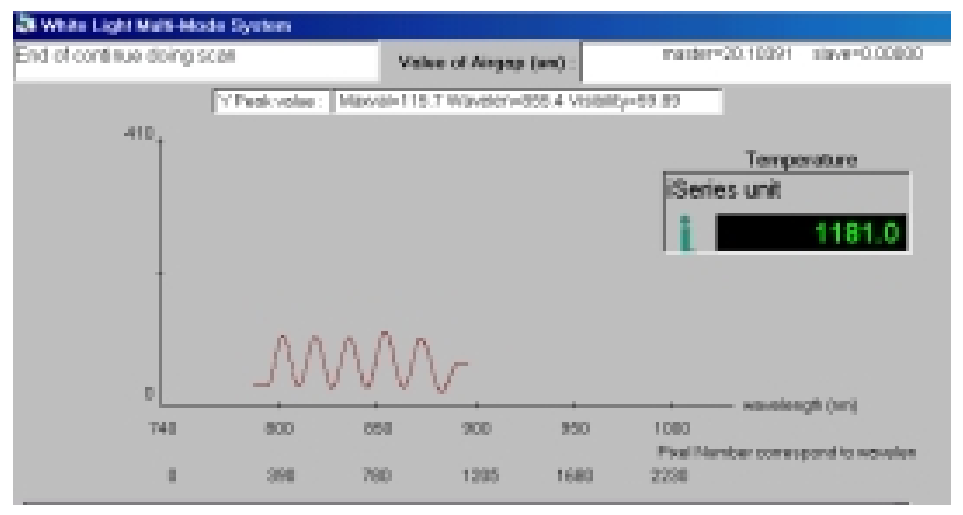

Figure1. Real time temperature is related to gap values

\section{Experiment setup optimization}

With a single-crystal sapphire disk as the sensing element, a Broadband Polarized-Light Interferometric (BPLI) high temperature sensor is presented in last report. The state of polarization of the broadband incident light is modulated by the high birefringence of the sapphire disk and becomes a wavelength-encoded signal, which is detected by an Optical Spectrum Analyzer (OSA) and then is processed by a computer, an internally developed algorithm is employed to directly calculate gap changes between two optical path between two orthogonal linear polarizations of light in a sapphire phase retarder, its phase retardation changes with temperature. The great advantages of this sensor are its simplicity and long-term stability in harsh environment.

A Broadband Polarized-Light Interferometric (BPLI) high temperature sensor's sensing head design scheme is shown in Figure 2. With a broadband Light Emitting Diode (LED) as the light source, a sapphire birefringent disk (90 degree orientation, i.e C-axis is perpendicular to surface) is sandwiched between parallel polarizers, with its principle axis (fast or slow axis) orientated at 45 degree to the polarization direction of the incident linearly polarized light. The transmitted interferometric spectrum signal can be expressed as :

$I(\lambda)=2 k I_{s}(\lambda)\left(1+\cos \left(\frac{2 \pi d(T) \Delta n(T)}{\lambda}\right)\right)$ 
Where $I_{s}(\lambda)$ is the spectral power distribution function with wavelength $(\lambda)$ of broadband light source; $k$ is a parameter describing the power loss of the optical system, and can be treated as a constant; $\mathrm{d}$ is the thickness of the sapphire disk; and $\Delta n=n_{e}-n_{o}$ is birefringence of the sapphire disk. Both $\mathrm{d}$ and $\Delta \mathrm{n}$ are functions of temperature $\mathrm{T}$. Equation (1) can be normalized with respect to $I_{s}(\lambda)$, the spectral power distribution function of light source, ideally, the interference fringes form a perfect cosine curve:

$I(\lambda)=2 k\left(1+\cos \left(\frac{2 \pi d(T) \Delta n(T)}{\lambda}\right)\right)=2 k\left(1+\cos \left(\frac{2 \pi f(T)}{\lambda}\right)\right)$

Where,

$f(T)=\mathrm{d}(\mathrm{T}) \Delta \mathrm{n}(\mathrm{T})$,

According to equation(2) the algorithm used to calculate $f(T)$ from transmitted interferometric spectrum is described as following:

The normalized transmitted interferometric spectrum, which is supposed to be a perfect cosine curve, consists of a series of maxima at certain wavelengths, if the wavelengths of two consecutive peak points are $\lambda_{\mathrm{i}}$ and $\lambda_{\mathrm{i}+2}, f(T)$ can be then calculated by equation:

$f(T)=\lambda_{i} \times \lambda_{i+1} /\left(\lambda_{i}-\lambda_{i+1}\right)$

All $f(T)$ are treated as gap values, which represent the optical path difference between two orthogonal linearly polarized light propagated in the sapphire disk. These gap values are related to temperature by calibration from room temperature to 1600 degrees Celsius or even higher.

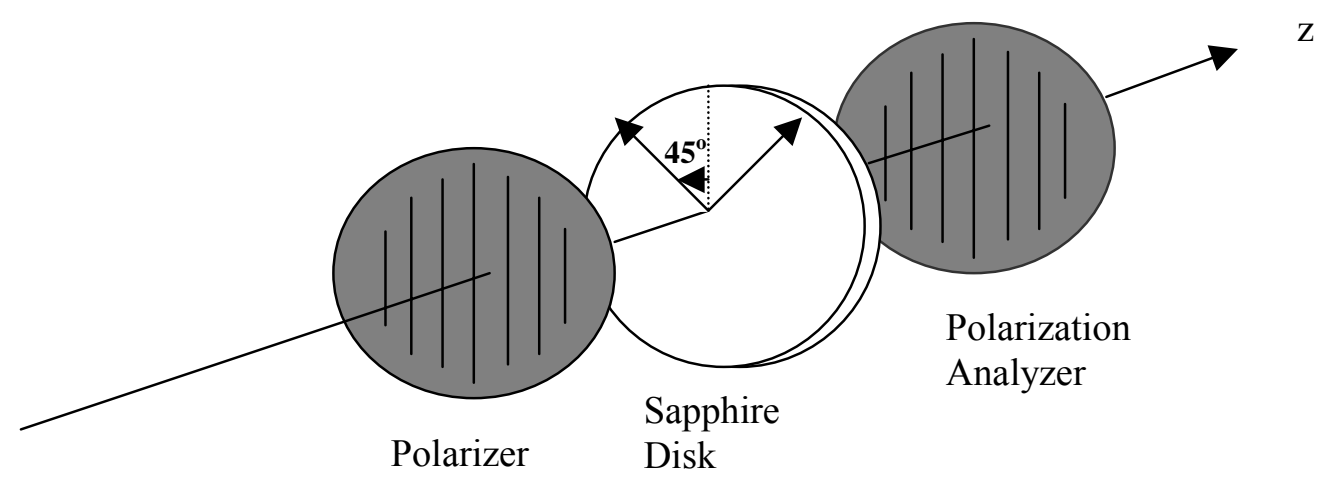


Figure 2. Sensing head design scheme: Polarized-Light Interferometry

The experiment setup in last report is employing a transmission mode, its setup is similar to the configuration shown in Figure 2, which means that there are two polarizers and two fiber collimators, which are located at two different sides of the sensing element (sapphire disk), one of them input light into the sensing element and the other collect output light, then conduct those light into OSA for analysis. This kind of setup works well if the high temperature field is limited in a small volume, such as in a small furnace used in last report, but for real coal gasifier, it is hard or impossible to use two fiber collimators at two ends. Based on the same working principle shown in Figure 2, reflection mode setup is used instead of transmission mode setup, where only one fiber collimator is used, it is used for both input light and also collecting light that reflected back from a 45-45-90 degree prism. Three different sensing head prototypes were designed based on commercial available products, as shown in Figure 3(a) (b) and (c) seperately.

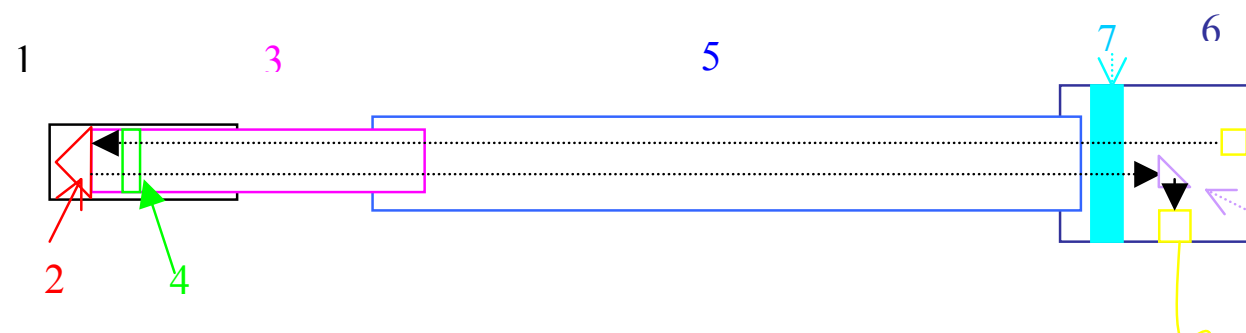

(a) Structure of prototype 1,

Components:

1, Cover tube : sapphire tube

2, Zerconia prism

3 , Sensing element holder: sapphire tube

4, Sapphire disk (orientation 90 degree, thickness 0.1 inch)

5, Extension tube: ceramic tube

6 , Collimator and polarizer holder, it may be a metal box 
7, Polarizer ( also work as analyzer)

8, Input collimator and fiber

, Output collimator and fiber

$10,90^{\circ}$ glass reflection Prism

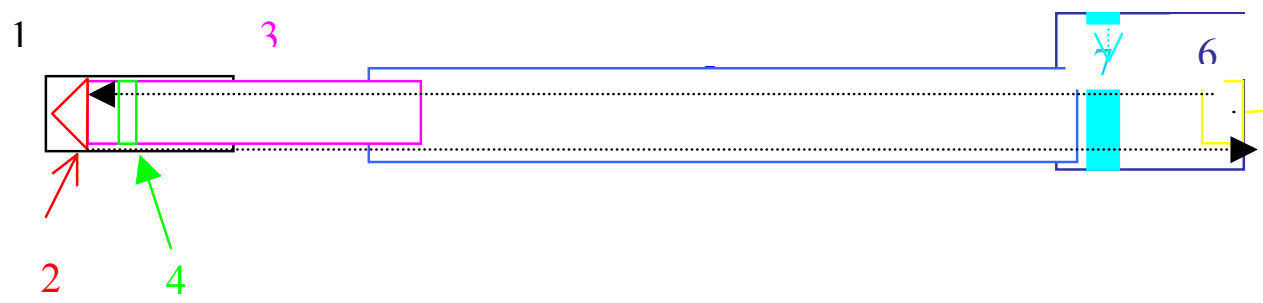

(b) Structure of prototype 2,

Components: one dual-fiber collimator replaces part 8,9, and 10 in prototype (1).

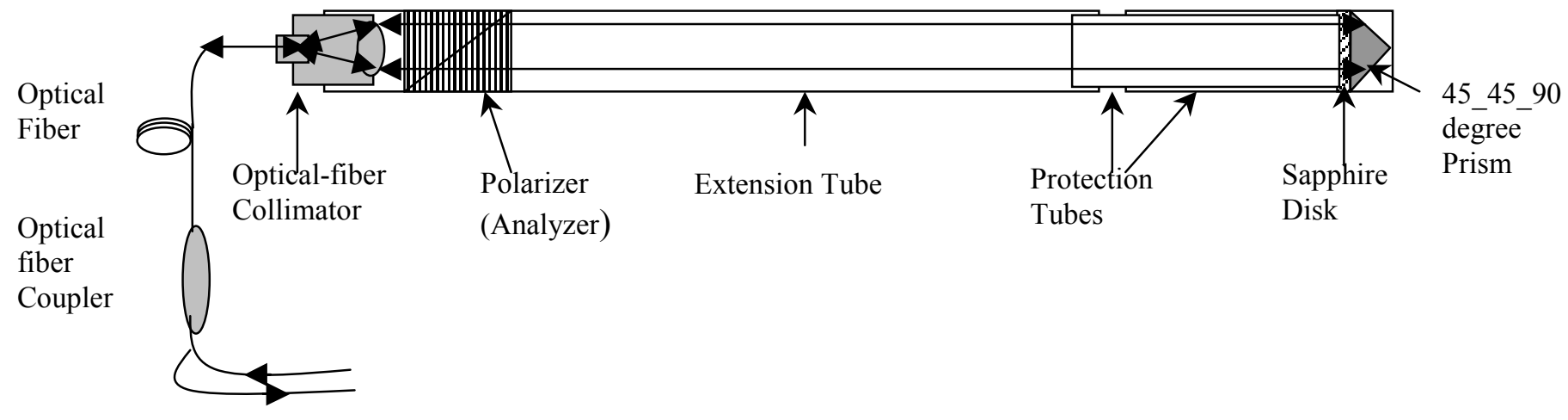

(c) Structure of prototype 3

Figure 3. Sensing tube prototype designs

\section{Available products for sensing tube fabrication:}

1, Commercial available cover tubes : sapphire tube

1. $1.0 "(25 \mathrm{~mm})$ OD* $0.051 "(1.3 \mathrm{~mm})$ wall*12.0"(30mm) long

2. 1.168 " OD* $0.062 "(1.5 \mathrm{~mm})$ wall*10.0" long $\$ 750.00$

2, Zerconia prism (can be polished by ourselves)

3, Sensing element holder: sapphire tube (commercial available) 
4, Sapphire disk (orientation 90 degree, thickness 0.1 inch, commercial available)

5, Extension tube: ceramic tube (commercial available)

6, Collimator and polarizer holder, (commercial available)

7, Polarizer ( also work as analyzer)( commercial available)

, Input collimator and fiber (commercial available)

, Output collimator and fiber (commercial available)

$10,90^{\circ}$ glass reflection Prism (commercial available )

By comparing prototypes in Figure3, structure (c) was selected because of its optical alignment simplicity and stability. This prototype sensor is fabricated as total sensing tube length about 2 meters, with diameter $3.5 \mathrm{~cm}$, which consists sapphire protection tube and extension tube ( round single bore cast alumina 99.8\% tube). Sensing element (a sapphire disk) is located in the protection tube which will be put into high temperature furnace, while at relatively low temperature end, both optical polarizer and optical fiber collimator are inserted into the tube. Since one 45-45-90 degree prism is used to reflect light back from the end of sensing tube, only one optical polarizer is used to work both as polarizer and polarization analyzer, which not only reduces the cost, but also simplifies the tube assembly. The total sensing tube is integrated as one fixed long tube, which makes it robust enough to survive harsh environment.

Possessing a high melting temperature (over $2000{ }^{\circ} \mathrm{C}$ ), chemically inert single-crystal sapphire is an attractive candidate to be employed under adverse conditions, such as organic solvents and acids, at elevated temperatures and chemically corrosive environments. In this sensor design, single crystal sapphire tube is employed to protect sensing element from corrosive high temperature hash environment, and zirconia's 4545-90 degree prism is used to reflect light back in the sensing tube, because of its cubic inner structure there is no birefringence in zirconia material, thus it will not affect temperature encoded optical signal generated by birefringence in sensing element. Photograph of zirconia prism and sensing elements( sapphire disk) is shown in Figure 4, and Figure 5 shows sapphire tubes: inner sapphire tube is used to support sensing element and zirconia prism, outer tube is used for protection. And Figure 6 is part of sensing head, 
it assembles all tubes, sensing element and zirconia prism together and will be put into high temperature enviroment.

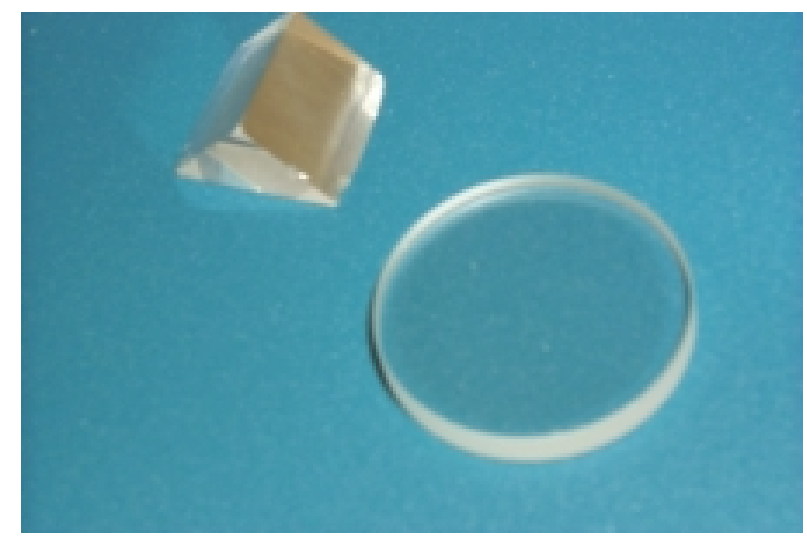

Figure 4. Zirconia prism and sapphire disk used in the sensing tube.
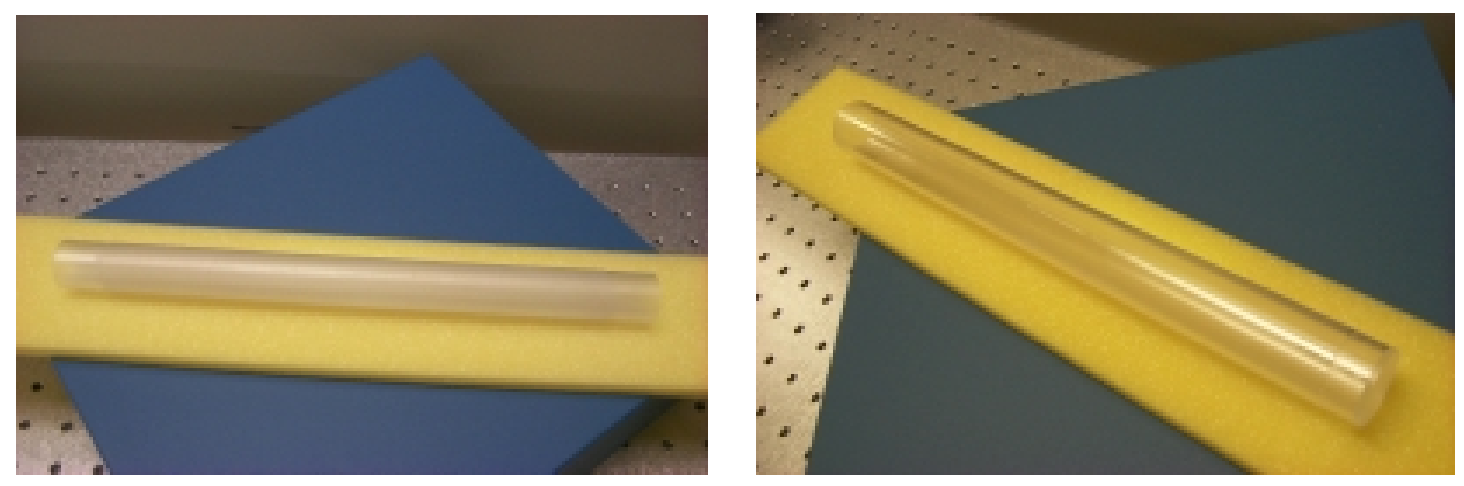

(a) inner tube

(b) outer tube

Figure 5. Sapphire tube used in the sensing tube

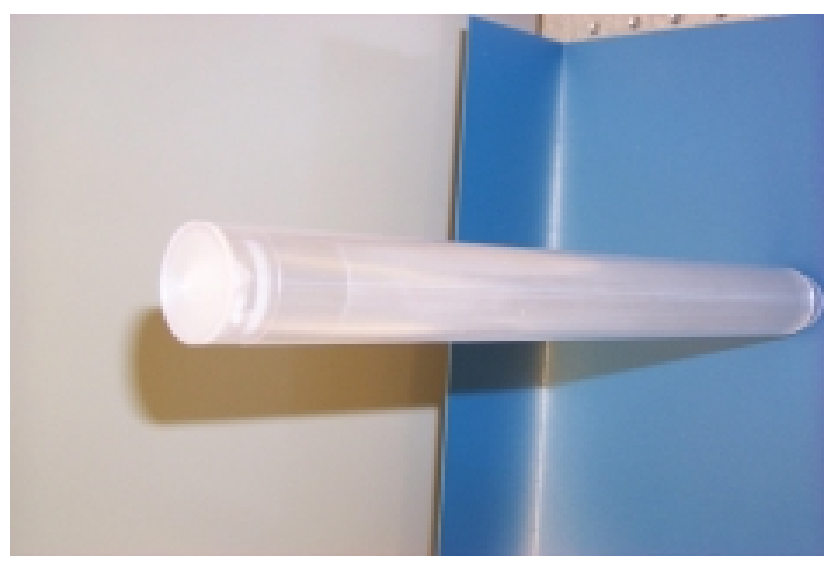

Figure 6 Part of sensing head 
Figure 7 shows configurations of the sensing system. Optical signal from a broadband LED (centered at $850 \mathrm{~nm}$, wavelength ranges from $810 \sim 890 \mathrm{~nm}$ ) is injected one $2.0 \mathrm{~m}$ lead-in multimode optical fiber cable and propagates to the sensor tube, the light coming out from fiber is collimated by a collimator and then pass through the polarizer, after the polarizer, the light becomes linearly polarized and is incident into the sensing elements perpendicularly, since one 45-45-90 degree prism is behind the sensing elements, light will be reflected right back, and passing through polarization analyzer, then be collimated into fiber and conducted into a fiber optic PC plug-in spectrometer (PC2000 from Ocean Optics, Inc.), which is composed of a grating and CCD array, the intensities of the spectral components of the signal are measured by the CCD array and further signal processing, such as demodulation and gap calculation, will be done in the computer. An Omga type B high-temperature thermocouple $\left(6 \% \mathrm{RH}-\mathrm{Pt}\right.$, resolution $0.5{ }^{\circ} \mathrm{C}$ under $1000{ }^{\circ} \mathrm{C}$ and $1.0{ }^{\circ} \mathrm{C}$ above $1000{ }^{\circ} \mathrm{C}$ ) was used as the temperature monitor; the sensor was heated in one high temperature furnace. Figure 8 shows the sensing system from different view angles.

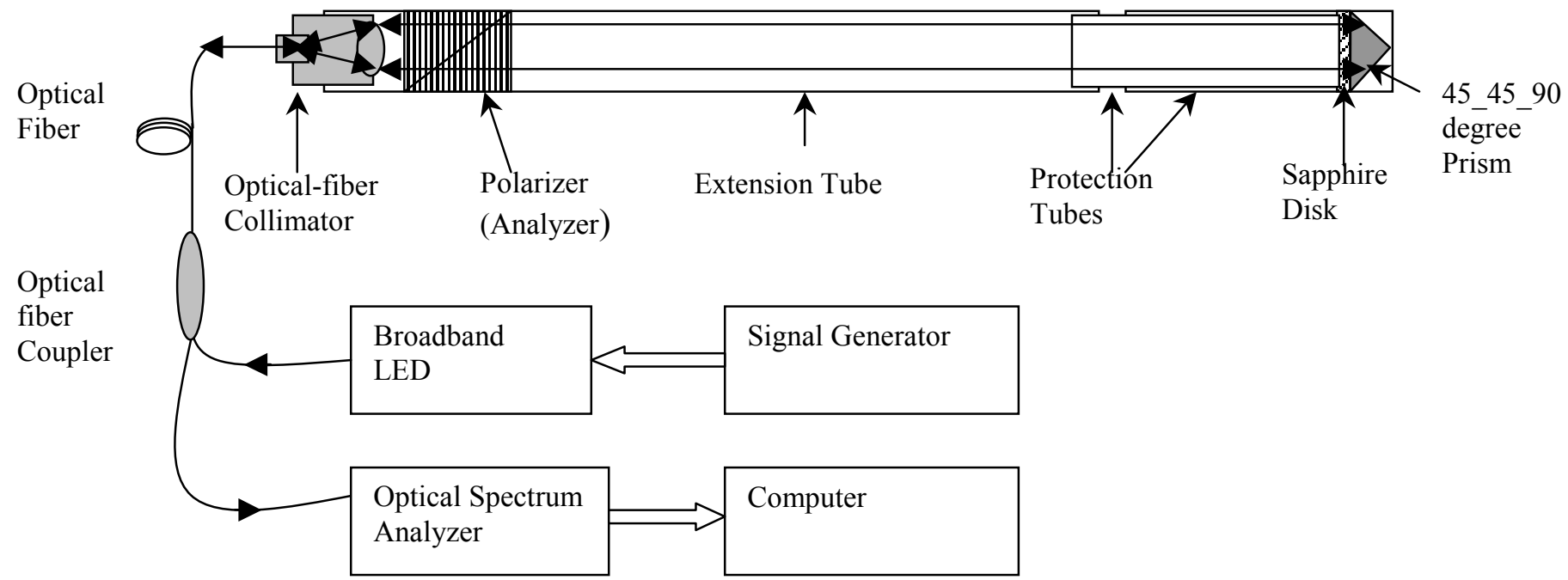


Figure 7. Configuration of the sensing system
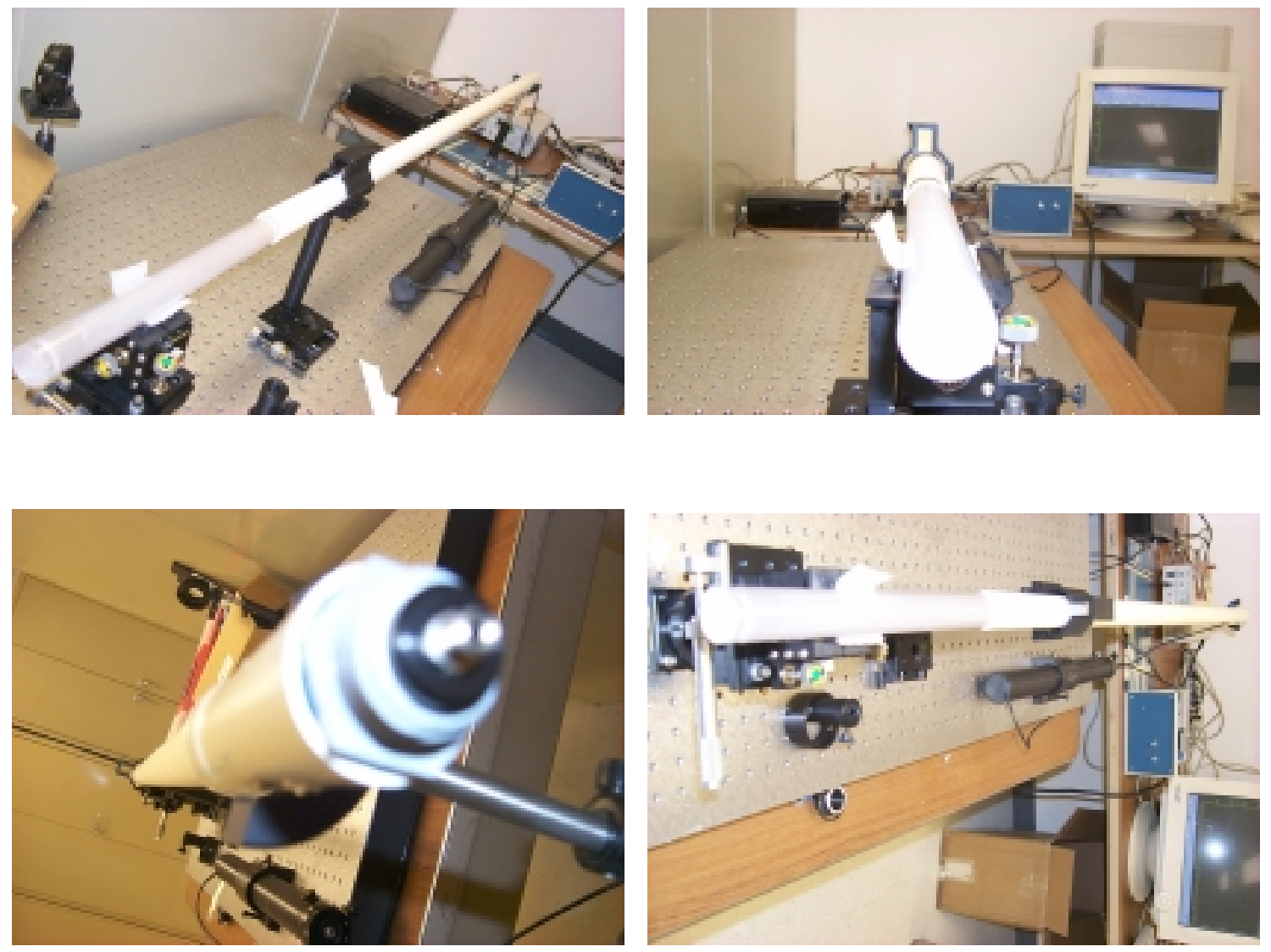

Figure 8. Different view angles of the sensing tube

\section{Optical source modulation to reduce blackbody radiation}

For the broadband LED emitting wavelength range $(810 \sim 880 \mathrm{~nm})$, the blackbody radiation becomes so strong for temperatures higher than $1000{ }^{\circ} \mathrm{C}$ that the background noise level will deform the interferograms formed by LED output light. At different temperatures, detected signals are shown in Figure 9, which contain two parts, one is the blackbody radiation background, and the other part is the interferogram generated by LED output light. Comparing figure (a) and (b) in Figure 9, it is obviously that interference fringes will be lifted up by blackbody radiation background when the 
temperature increases (from $1450{ }^{\circ} \mathrm{C}$ to $1600{ }^{\circ} \mathrm{C}$ shown here), and the theoretical blackbody radiation curve is shown in Figure 10.

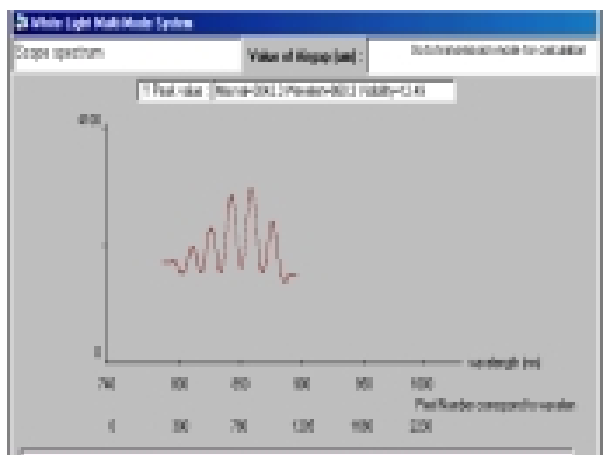

(a) @ $1450{ }^{\circ} \mathrm{C}$

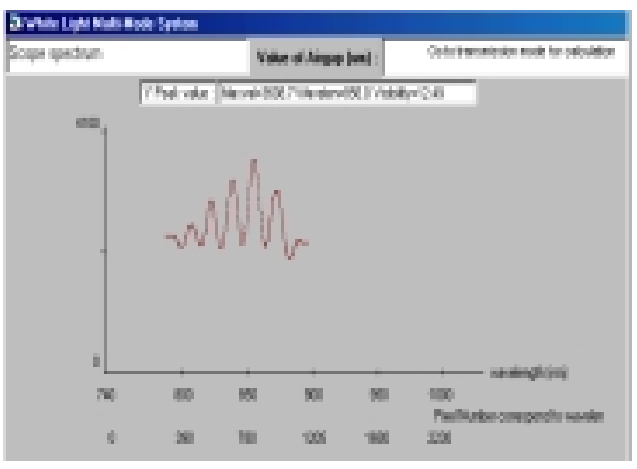

(b) @ $1600{ }^{\circ} \mathrm{C}$

Figure 9. Optical signals detected by OSA at different Temperature.

In order to minimize the influence of the blackbody radiation background, the LED output light is modulated, in last report, certain frequency sinusoidal signals are used to modulated LED output light. Since blackbody radiation is only a DC component in detected signals from spectrometer, it can be filtered out with a high-frequency band-pass filter. i.e. by modulating LED output light with certain frequency sinusoidal signals, and combining with digital signal filter (IIR filter, Infinite-Impulse Response filter), background from blackbody radiation can be reduced, especially in high temperature circumstance. While an IIR filter can only be implemented in a computer with certain accuracy, to implement an ideal IIR filter is impossible, so signals after digital filter will be deformed.

A new modulation signal: rectangular waveform signal is employed as an alternative modulation signal source to sinusoidal waveform signals, which even makes demodulation process much easier than sinusoidal waveform modulation method. By selecting the amplitude of rectangular modulation signal, LED output light will be alternated between 0 and certain constant power, when its output light is 0, OSA only detect the blackbody radiation background intensity and then is stored in the computer, when its output is certain constant power, OSA will detect not only blackbody radiation 


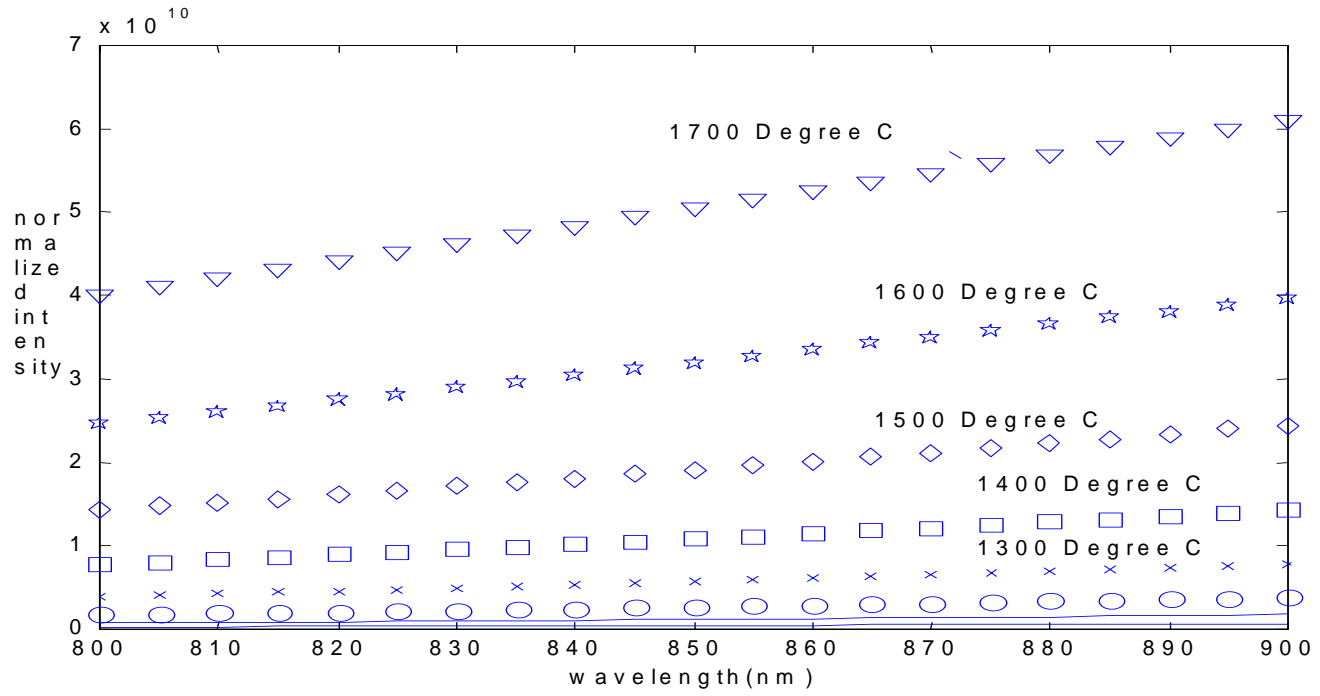

Figure 10. Theoretical results of blackbody radiation at different temperatures for wavelength range (800 900nm)

background, but also detect the interferogram generated by LED output light, this part will contain the temperature information we needed, and it can be purified by subtracting the blackbody radiation part that is already stored in the computer. Noise from blackbody radiation can be highly reduced after demodulation on received signals by this kind of signal processing method, which can seen in figures shown in next session.

\section{Experiment results and discussion}

The photographs of the sensor system are shown in Figure 11. 


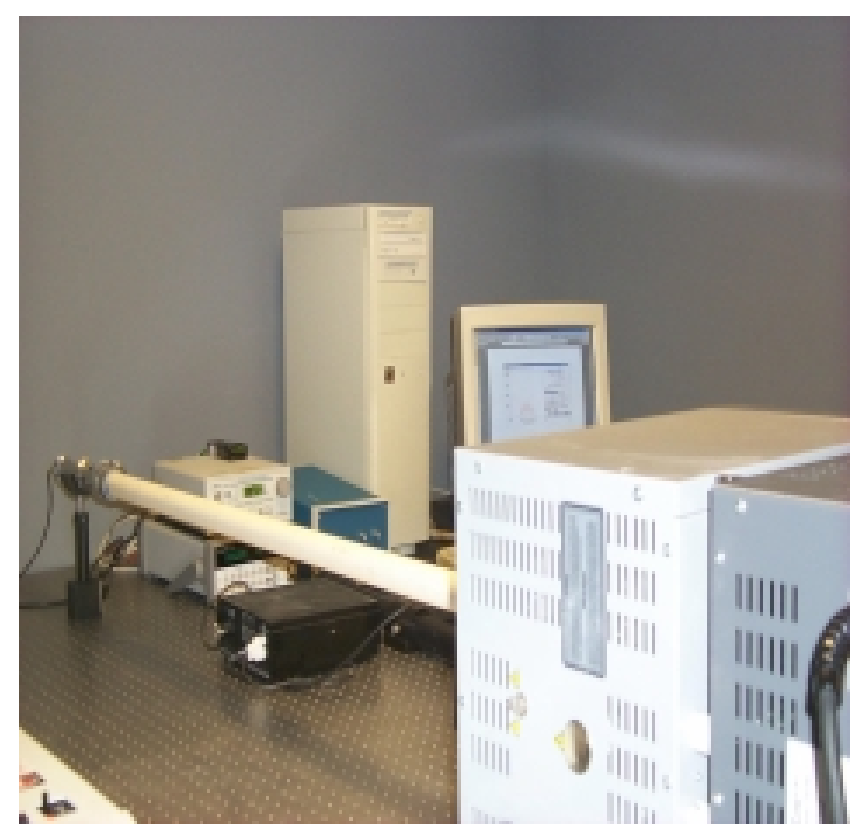

(a) Overview of the sensing system

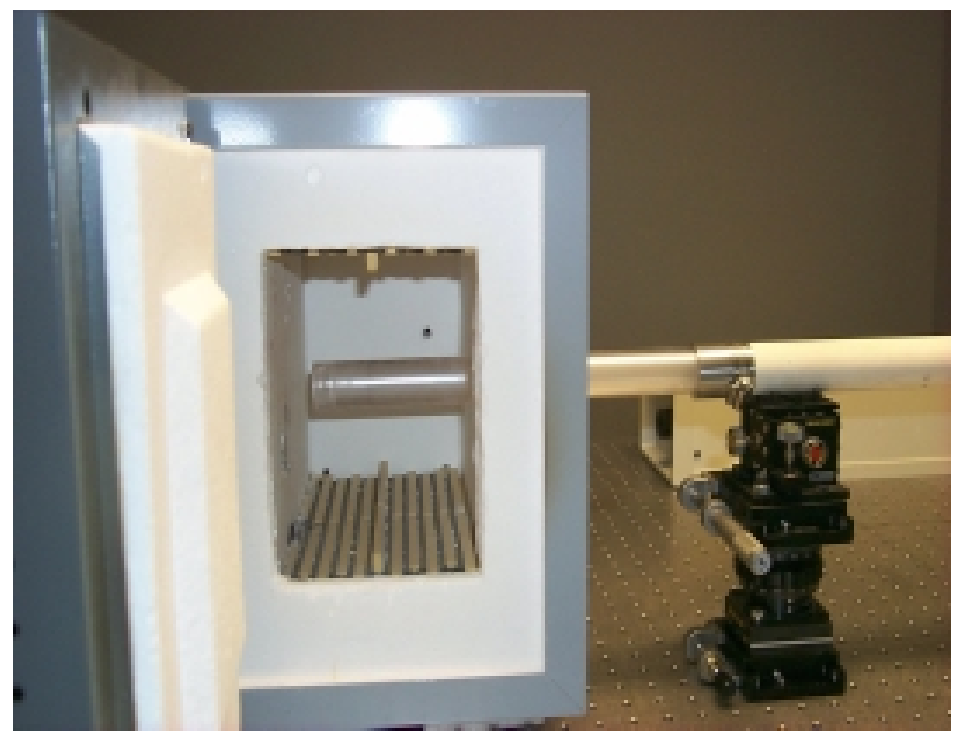

(b) Sensing head in the high temperature furnace 


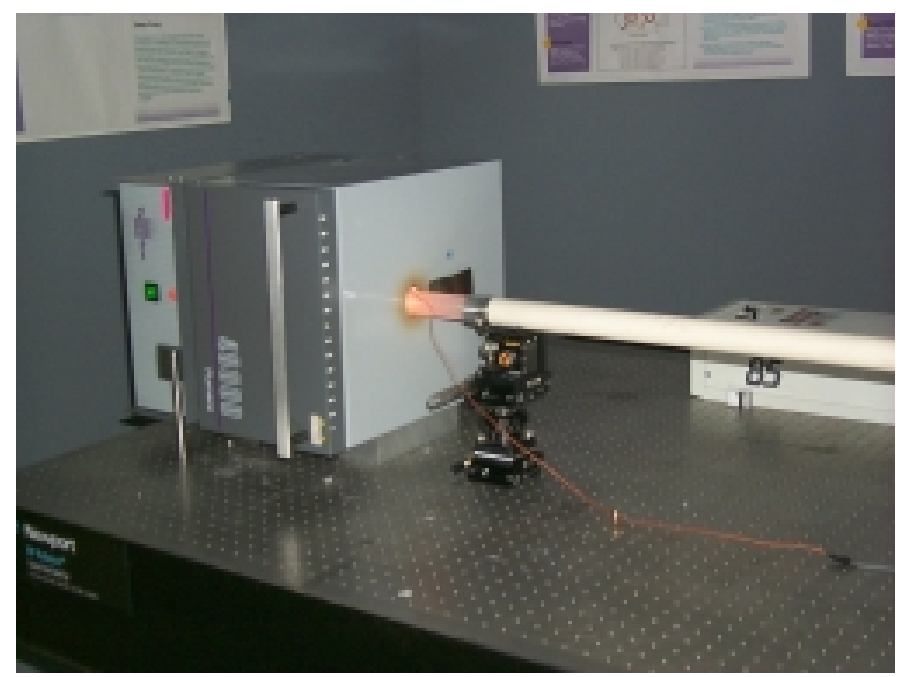

(c) sensing head is heated up over $1000{ }^{\circ} \mathrm{C}$

Figure 11. Sensing system setups

Typical output light signals are shown in Figure 12. Even with different blackbody radiation background at different temperatures, they appear to be similar in intensities, obviously blackbody radiation is reduced, which make the measurement temperature can be upto $1600{ }^{\circ} \mathrm{C}$ or even higher. According to Equation (3), gap values (shown in figures as airgap) were calculated. Figure 13 shows a gap values vs. temperature curve, which indicate the one-to-one relation between gap value and real time temperature without any jump occurred in last report.
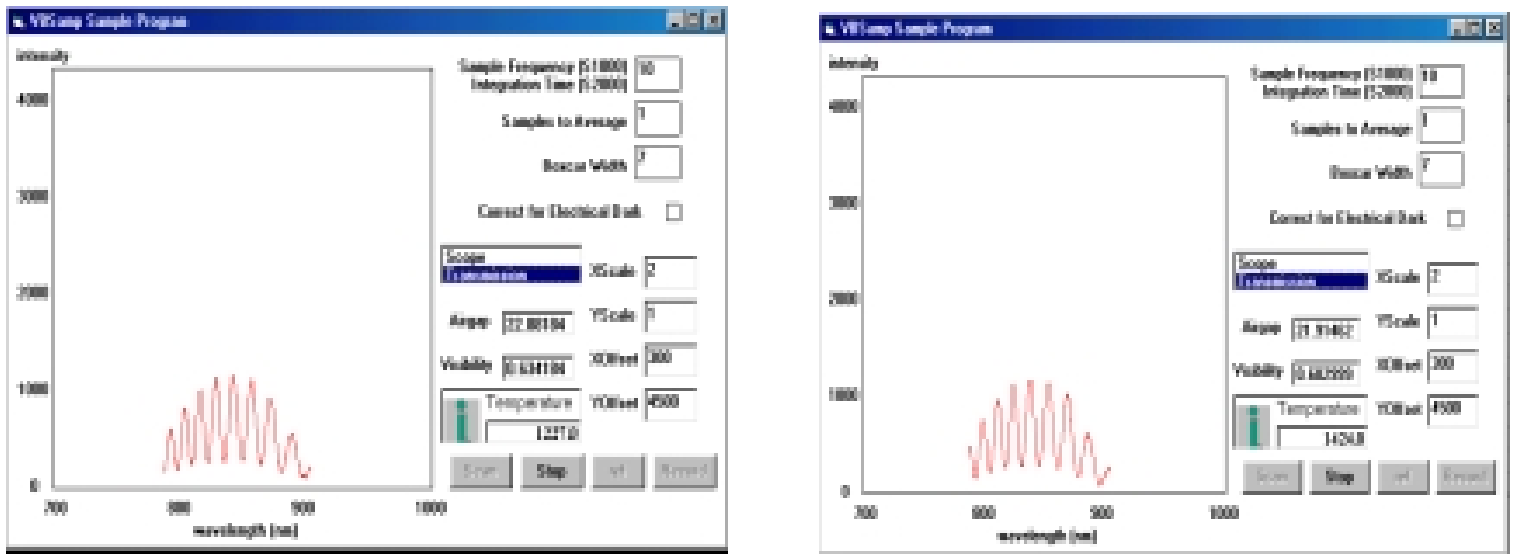
(a) $@ 1227.0^{\circ} \mathrm{C}$

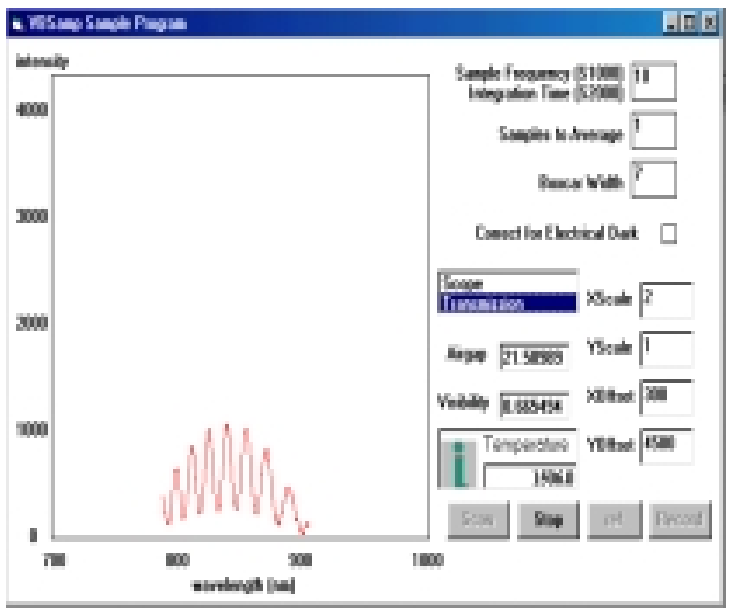

(c) $@ 1506.0^{\circ} \mathrm{C}$ (b) @ $1424.0^{\circ} \mathrm{C}$

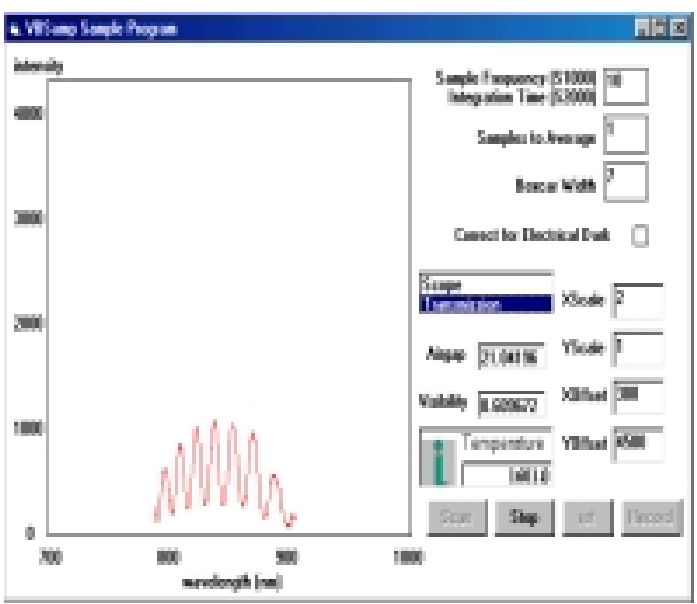

(d) $@ 1601.0^{\circ} \mathrm{C}$

Figure 12. Typical output light signals at different temperature

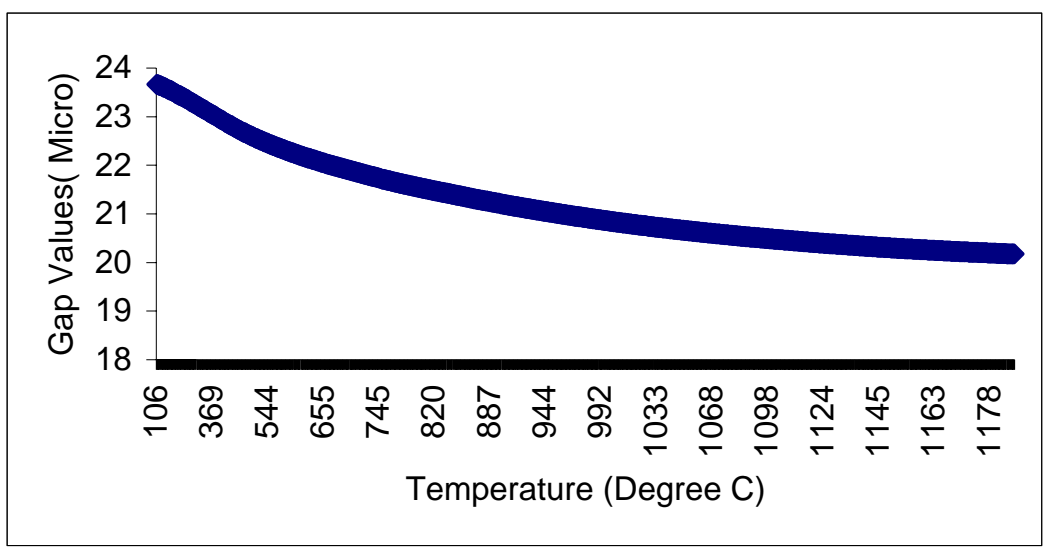

Figure 13. Gap values Vs. temperature curve

According to Equation (3), gap values are one by one related to real-time temperatures, and different sensing elements with various thicknesses needs to be calibrated to get its own gap vs. temperature response curve. To get the calibration curve, furnace was kept at certain temperature for a period of time, then the gap values should be stable during that period of time, that is what shown in Figure 14. 


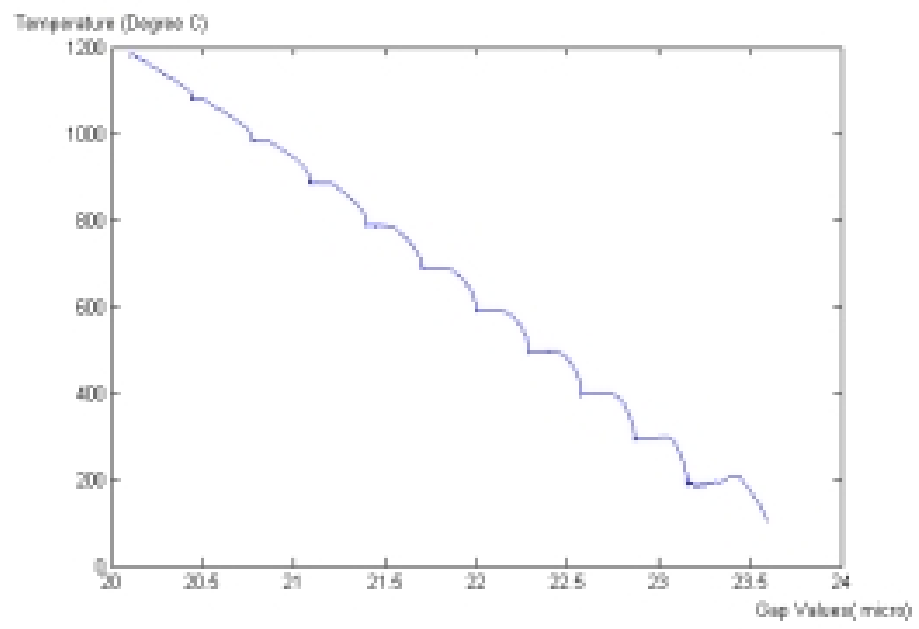

Figure14. Calibration measurement process

Every $50^{\circ} \mathrm{C}$, gap values are collected for calibration, these results show linear relationship (shown in Figure15) between temperature and gap values, which is not accurate, bigger errors are generated compared thermal couple output and the designed sensing system output, as shown in Figure 16 for the measurement process shown in Figure 13.

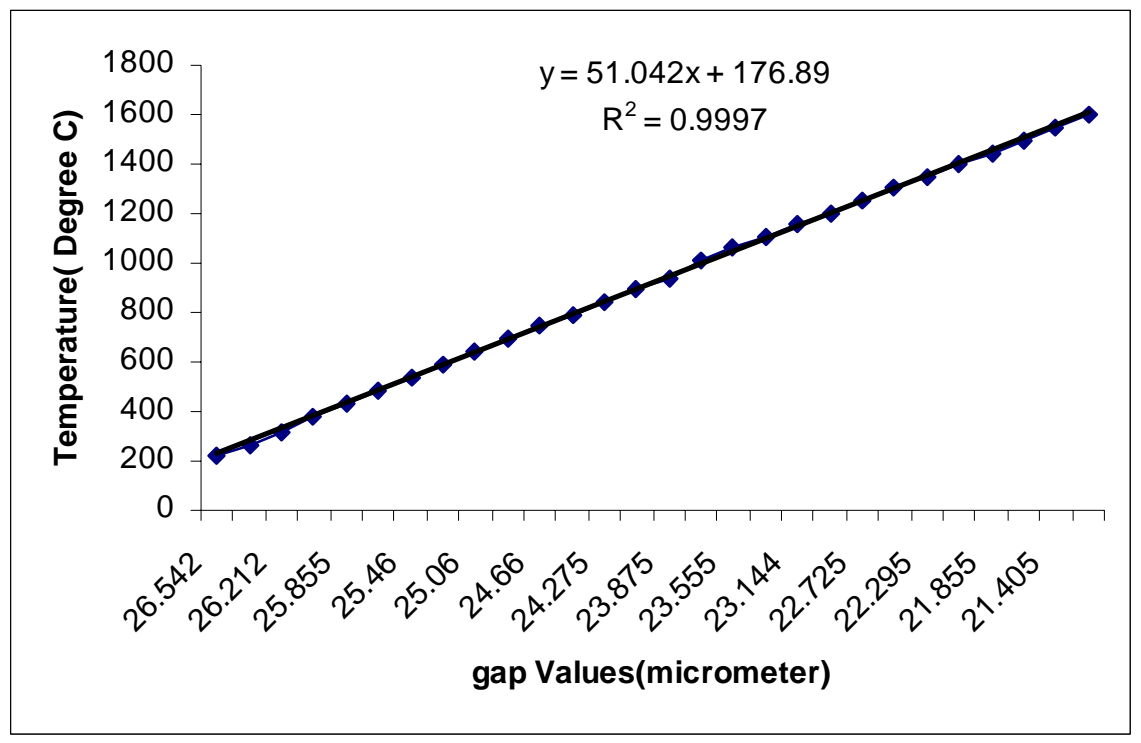

Figure15 Calibration curve between temperature and gap values 


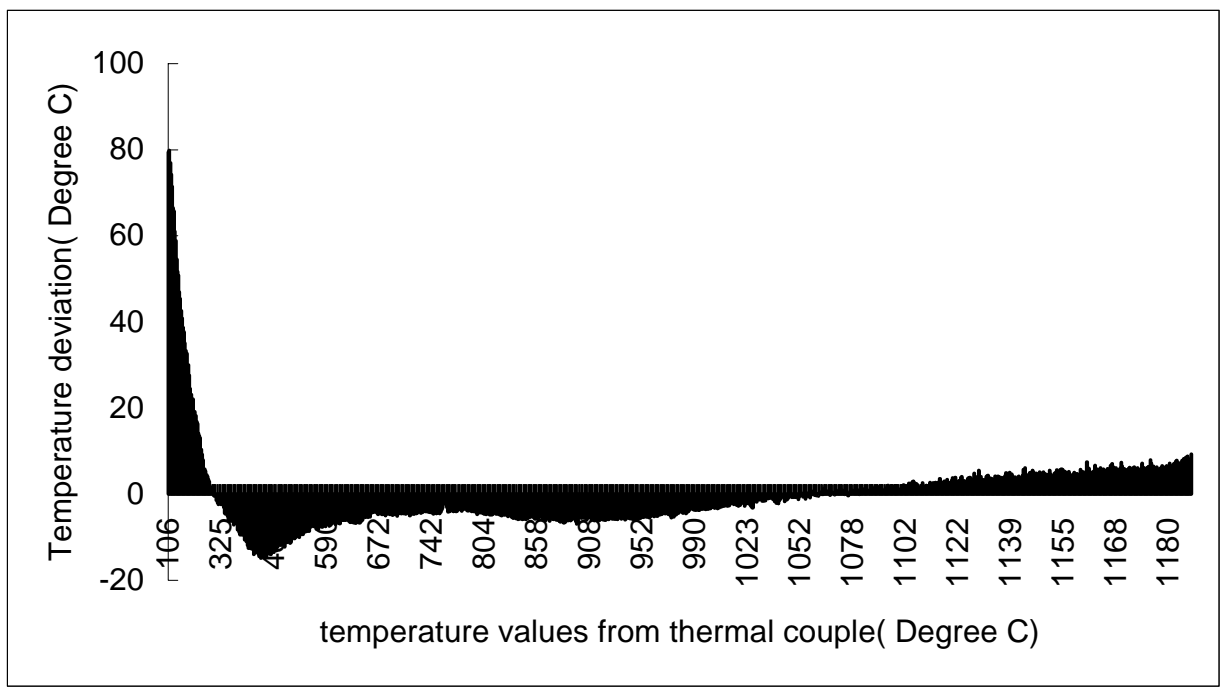

Figure 16. Error results with linear calibration curves (y-axis is deviation between sensing system output temperature values and thermal couple output)

Figure 17 shows another calibration curve for a sapphire disk (0.98inch diameter and 0.13inch thickness). The data was obtained every $50{ }^{\circ} \mathrm{C}$ for the range from $300{ }^{\circ} \mathrm{C}$ to $1500^{\circ} \mathrm{C} . \mathrm{A} 4^{\text {th }}$ order polynomial was used to do curve fitting instead of the one-order linear equation. The polynomial equation is shown in the upper figure, and the figure below shows the temperature deviation from the curve-fitting results and the experimental measurement results of calibration process. The measurement with the same sapphire disk was repeated three times as shown in Figure18. The repeatability between the temperature and gap values is very good. To determine the performance for these three different measurement processes, each of these curves are evaluated separately by a calibrated polynomial equation obtained as in Figure17. The temperature deviation of each curve from the calibrated results is shown in Figure19, which means that assuming type B thermocouple measurement results are absolutely accurate and the curve-fitting equation is exactly right, all of these three measurements have errors in the range of \pm 4 
${ }^{\circ} \mathrm{C}$ (accuracy $0.27 \%$ for $1500{ }^{\circ} \mathrm{C}$ ) for a temperature range higher than $300{ }^{\circ} \mathrm{C}$. For a temperatures lower than $300{ }^{\circ} \mathrm{C}$, one temperature curve shows errors of about $12{ }^{\circ} \mathrm{C}$.

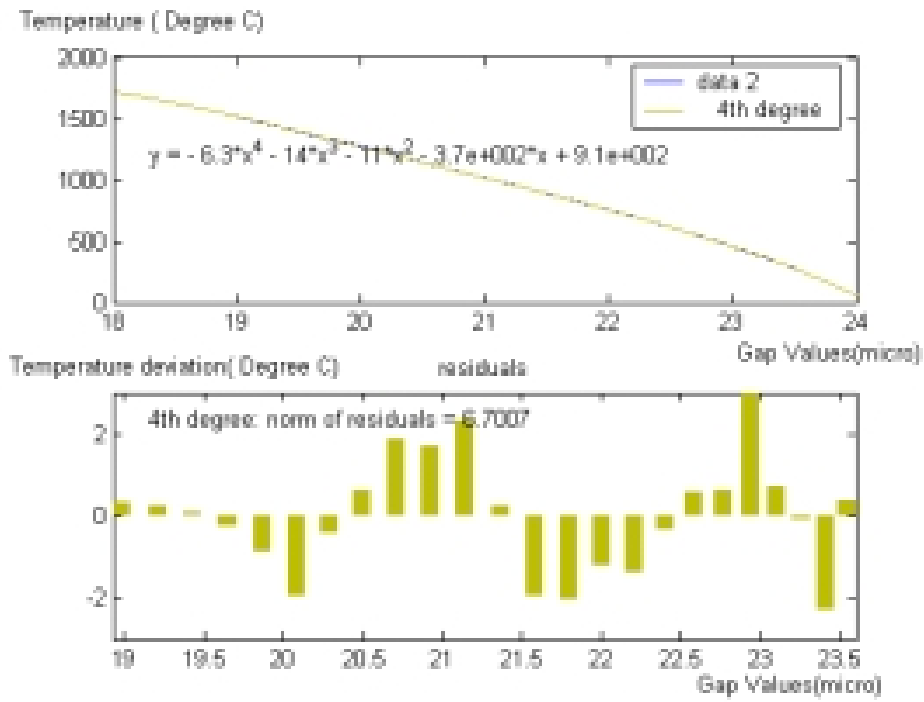

Figure 17. Curve-fitting for calibrated data for sapphire disk with thickness 0.13 inch.

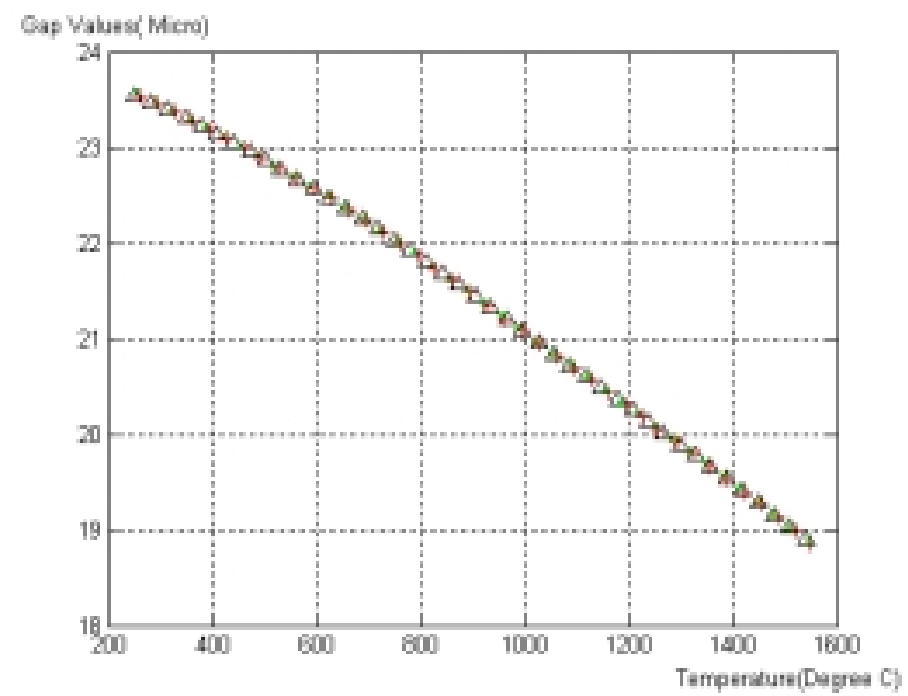


Figure18. Relationship between temperature and gap values measured three times with a sapphire disk with thickness 0.13 inch. The three different processes are represented by $\bullet, \Delta$, and + respectively.

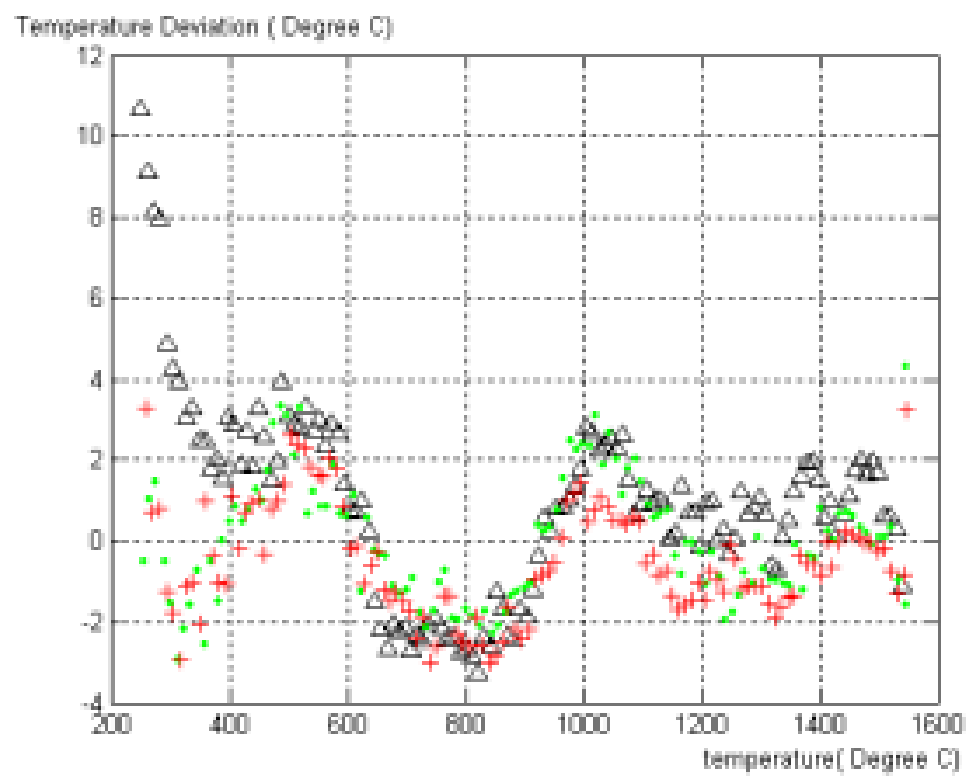

Figure19. Temperature deviation evaluation for each curve in figure6 with calibrated equation from figure 5 . Three different process represented by $\bullet, \Delta$, and + respectively. 


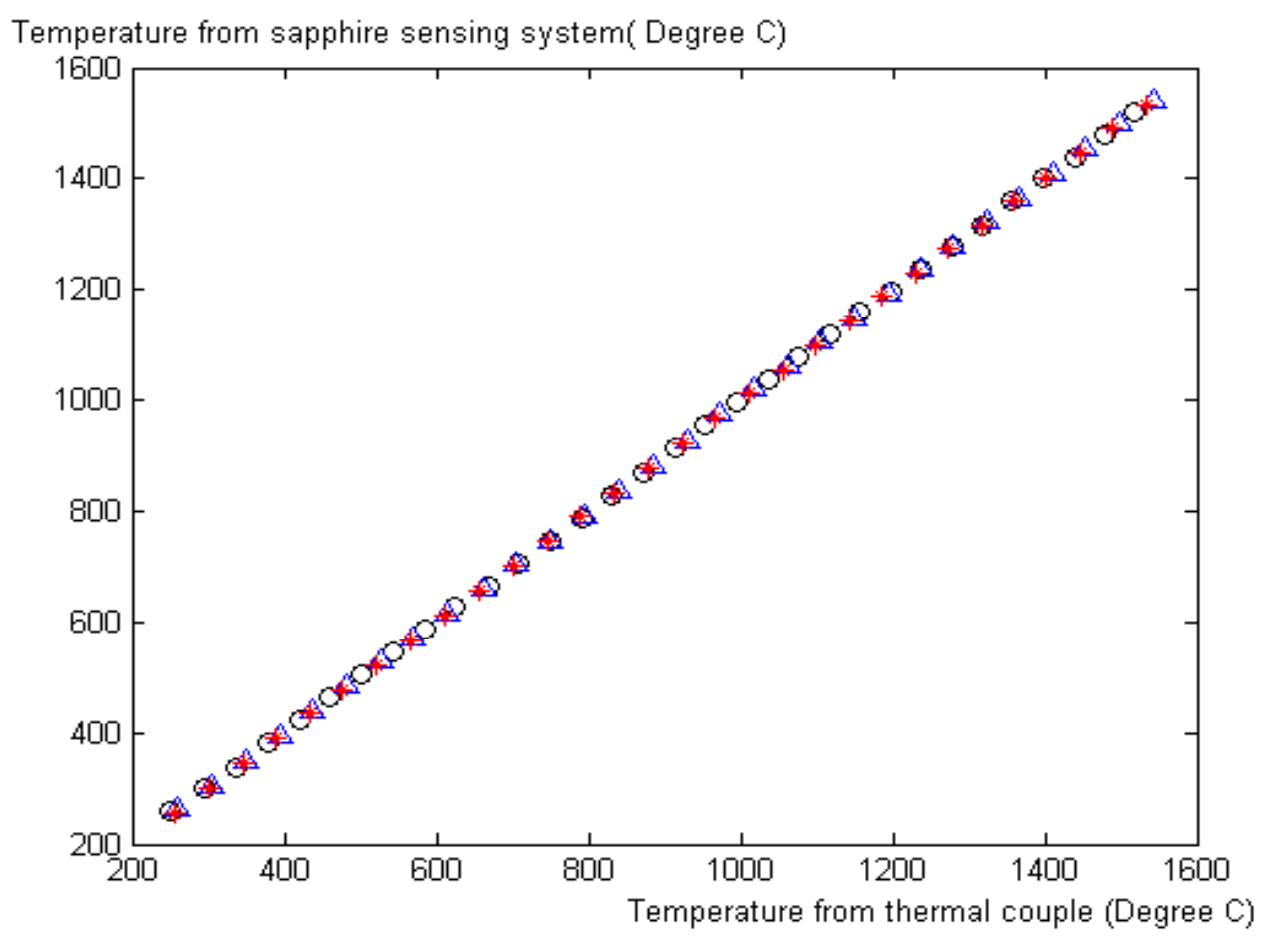

Figure 20. Relationship between thermal couple measured temperature and sapphire sensing system output temperature.

Further analysis on the three repeated measurement curve results are performed as shown in Figure 20. This figure shows the relationship between the thermocouple measured temperature and the sapphire sensing system output temperature. The ideal curve will be a straight line crossing the origin with a slope value of 1 . If the sapphire sensing system output is equal to the thermocouple output, as shown in the figure, the straight line is very close to the ideal line, which means that the accuracy of the designed sapphire sensing system is at least comparable to B-type thermal couple.

Also, according to experimental results, the relationship between the gap values and the temperature is not linear, which means that the relation shown in Equation (3) is not 
linear. This means that the result that both the thickness of the sapphire disk and birefringence of sapphire material are all functions of temperature. Also, according to Equation (3), the resolution for the sensor depends on the selected thickness of the sapphire disk. The thicker the disk is, the higher the resolution that can achieved. While the thickness is increasing, the interference fringes will become dense, which will be limited by the resolution of the OSA. Thus the resolution of the sensor depends on the resolution of the optical spectrometer. For the sapphire disk used in the three experiments discussed above, the gap resolution is $0.02 \mathrm{~nm}$, and for the whole measurement range $225 \sim 1580{ }^{\circ} \mathrm{C}$, the gap changed from 23.58 to 18.84 microns. The average gap change will be $0.035 \mathrm{micro} /{ }^{\circ} \mathrm{C}$, which means that the resolution is better than $1{ }^{\circ} \mathrm{C}$. This means that the resolution of this sensor system is at least comparable to that of B type thermocouple, if not better.

One problem that needs to be solved in the calibration process, i.e. for different measurement processes; there will sometimes be a constant shift for temperature between expected values and measurement values, as shown in Figure 21. In this figure, the triangle curve, star curve and circle curve represent three different measurement runs. Although, these measurements are all temperature increasing processes, the circle curve and the star curve repeated themselves quite well, while the triangle curve is about $65^{\circ} \mathrm{C}$ over the expected values for its measurement range, after shift $65^{\circ} \mathrm{C}$ down of its measured values, it overlaps with the other two curves, which is shown in Figure 22. These phenomena will affect the repeatability of the sensing system, which needs to be analyzed and solved in the future work. 


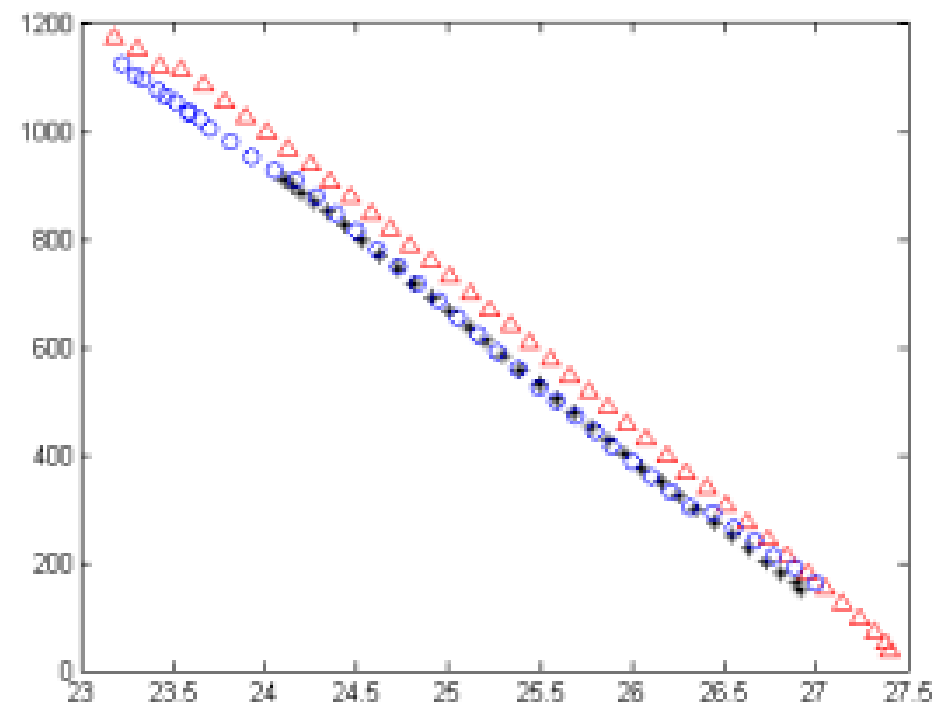

Figure 21. Three different measurement results for temperature increasing processes with sapphire sensor.

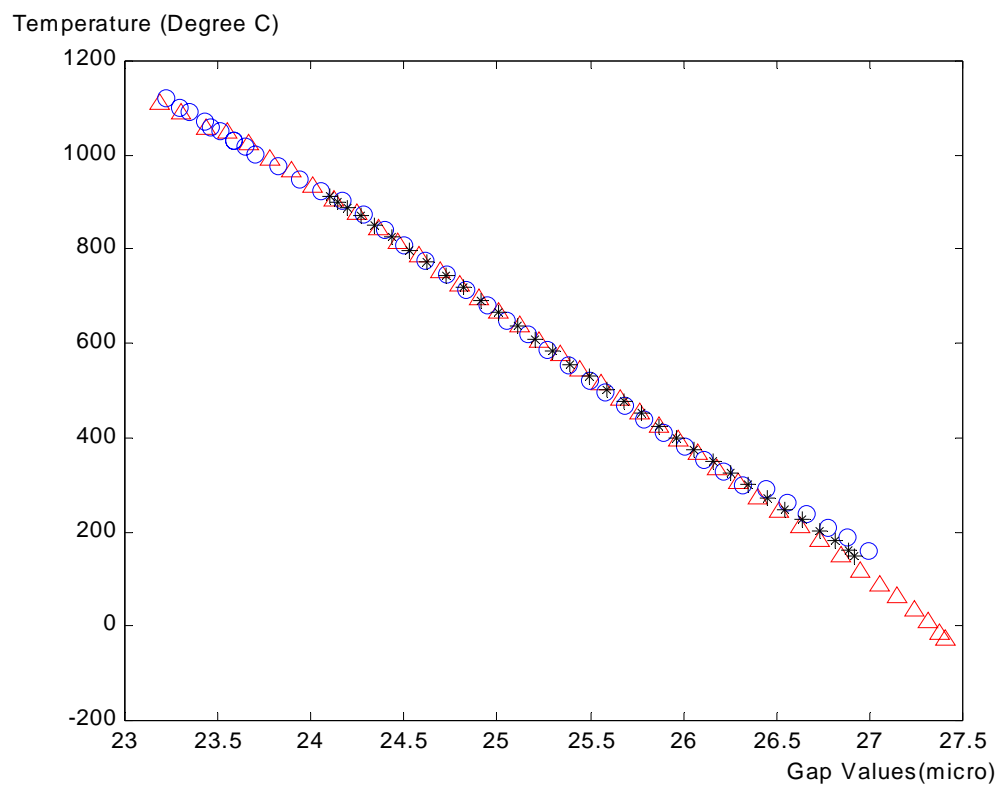

Figure 22, Temperature measurement curves after recalibration. 


\section{Conclusions and future research directions}

Improved versions of the Broadband Polarized-Light Interferometric Sensor (BPLIS)

high temperature sensor system has been designed and tested. With one single crystal sapphire disk with 0.13 inch thickness as the sensing element, the temperature sensor achieve a wide dynamic range over $1600^{\circ} \mathrm{C}$ with an accuracy better than $0.26 \%$, and resolution less than $1{ }^{\circ} \mathrm{C}$ of full scale. Its current resolution and accuracy are comparable to the B-type thermocouple. Further improvement on resolution can be done by changing the sensing element thickness. The structure of this sensor is simple and cost-effective for high temperature sensing, and is also designed to be robust enough to survive in real engineering environments.

Based on the results shown in this report, the future research work will mainly focus on three parts:

First, further calibration work will be done to reduce the problem mentioned in the last part of the last section. This problem may be solved by modify the algorithm that is used to do the signal demodulation and gap value calculation in computer.

Second, the sensor head will be fixed by using high temperature alumina cement. According to the sensor head design prototype, further optimization of the sensor head assembly will be tried, such that the sensor can survive high temperature hash environment. 
Third, the software design will be upgraded for the whole system. By employing Visual_BASic or LabVIEW; a graphical user interface (GUI) will be used to display the data for inspection and the data will also be stored on hard disk for further analysis. 